\title{
Giant field enhancement at carbon nanotube tips induced by multi-stage effect
}

\author{
J. Y. Huang, S. Chen, S. H. Jo, K. Kempa, and Z. F. Ren \\ Department of Physics, Boston College, Chestnut Hill, Massachusetts 02467
}

Recently we have reported an extremely strong field emission from carbon nanotubes (CNTs) grown on carbon cloth (CC), with the field enhancement factor $(\gamma)$ of up to $18,800 .{ }^{1}$ In this work we study the origins of this effect, by investigating field emission from individual CNTs grown on CC, in a transmission electron microscope (TEM) equipped with a piezo-driven scanning tunneling microscopy (STM) probe. ${ }^{2}$ Microscopic analysis reveals a multistage structure of some of the CNTs, characterized by an order of magnitude smaller CNTs branching-off the tips of bigger CNTs (or carbon fibers). We show that the multi-stage structure causes a macroscopic enhancement of the electric field, which can match that of a single macroscopically long CNT with length equal to the combined length of all stages, and the tip radius equal to that of the thinnest CNT in the structure. This explains the observed giant $\gamma$.

The multiwall CNTs were grown by chemical vapor deposition on CC. We found a significant fraction of the CNTs have a multi-stage structure, as shown in Fig. 1. This structure is characterized by an order of magnitude smaller CNTs, growing at the tips of larger CNTs or CC. Structure shown in Fig. 1 consists of three stages: a carbon fiber (Fig. 1(a)), a large CNT (Fig.1(b)), and a small CNT (Fig. 1(c)). The current-voltage (I- $V$ ) curves were taken for a CNT shown in Fig. 2(c), mounted on a gold rod shown in Figs. 2(a) and 2(b). The characteristic is comparable to that from individual CNTs studied in the scanning electron microscope (SEM). ${ }^{3}$

Fig. 3 shows of $\gamma-d$ plots for various CNTs. Fig. 3(a) shows data from two CNTs (A and B) with different radius of $10 \mathrm{~nm}$ and $22 \mathrm{~nm}$, respectively, in the small gap region, $d<5 \mu \mathrm{m}$. In this regime, $\gamma$ decreases linearly with $d$. Solid lines are linear interpolations, which show that the slope is equal to the inverse of the CNT radius. Fig. 3(b) shows data in the large range of $d$, for two similar CNTs: our CNT A (shown also in Fig. 3(a)), TEM picture of which is shown in Fig. 2, and the CNT C, taken from Ref. 3. In order to fit results for both CNTs into a single figure, we use scaled quantities $\gamma / \mathrm{M}$ and $d / \mathrm{M}$. We chose $\mathrm{M}=1$ for the CNT C, and $\mathrm{M}=30$ for the CNT A.

To interpret the above results, we have performed calculations of the local electric field, based on the field emission geometry shown in Fig. 4(a). The electrostatics yields the following simple formula for the local electric field at the top of the small sphere (CNT tip) $E=E_{0} \gamma$. With $\gamma=\frac{l}{D}\left(1+\frac{d}{r}\right)$ (Eq.1), where $E_{0}=V / d$ is the reference electric field. This formula is in excellent agreement with the experimental data in the entire range of $d$ values measured, as shown in Fig. 3. For $d<<D$ (small gap regime), Eq. 1 reduces to $\gamma=1+d / r$ (Eq.2), which is in excellent agreement with the experimental results, as shown in Fig. 3(a). Microscopic measurements of the CNT diameters agree very well with those obtained from the slopes of Fig. 3(a). For $d \approx D$ (large gap regime) Eq. (1) yields the well known approximate result $\gamma=l / r$ (Eq.3). The proportionality of $E$ to $E_{0}$ in our formalism implies that for a multi-stage structure, with each stage much smaller than the previous one $\left(l_{i+1} \ll<l_{i}, r_{i} \ll l_{i}\right.$, and $\left.l_{i+1} \ll r_{i}\right)$, the total $\gamma$ at the tip of the smallest stage, $\gamma_{m s}$, is a product of the $\gamma$ for individual stages. Thus, $\gamma_{m s}=\prod_{i=1}^{m} \gamma_{i}$ (Eq. 4), where $i$ is the stage index, $m$ is the total number of stages. $\gamma_{i}$ can be given by Eq. (3), i.e. $\gamma_{i}=l_{i} / r_{i}$.

Importance of the multi-stage enhancement is illustrated in Fig. 3(b). It shows the $\gamma$ - $d$ plots for two similar CNTs. CNT C has length of $3.2 \mu \mathrm{m}$, diameter of $17 \mathrm{~nm}$. Its aspect ratio is $l / r=188$, and this would imply (from Eq. 4) $\gamma \approx 188$. This is in approximate agreement with the value $\gamma \approx 230$, extracted from the experimental results, ${ }^{2}$ displayed also in Fig. 3(b) as squares. While the $\gamma$ of CNT C agrees roughly with Eq. 4, the one for CNT A, does not. This CNT has its $l=3 \mu \mathrm{m}$ and $r \approx 10 \mathrm{~nm}$ (see Fig. 2 (c)). Therefore the aspect ratio is about 300, but Fig. 3(b) clearly shows that the $\gamma$ for large $d$ exceeds 4000 (note, that $\mathrm{M}=30$ for this CNT), instead of saturating at approximately 300 . The reason for that is the multi-stage enhancement, $\gamma_{\text {tot }}=\gamma_{n t} \gamma_{\text {rod }}=300 * 24=7200$. Even larger $\gamma$ is expected for the multi-stage CNT system grown on CC as shown in Fig. 1 . Here $l_{1} \approx 300 \mu \mathrm{m}, r_{1} \approx 7 \mu \mathrm{m} ; l_{2}=7 \mu \mathrm{m}, r_{2} \approx 0.06 \mu \mathrm{m}$; $l_{3} \approx 1.6 \mu \mathrm{m}, r_{3} \approx 0.026 \mu \mathrm{m}$. Thus, from Eq. 4 the $\gamma_{\text {total }}=\gamma_{1} * \gamma_{2} * \gamma_{3} \approx 3 \times 10^{5}$, which explains the giant $\gamma$ obtained from the large area measurements. ${ }^{1}$

\section{References}

1. S.H. Jo, D.Z. Wang, J.Y. Huang, W.Z. Li, K. Kempa, and Z.F. Ren, Appl. Phys. lett. 85, 810 (2004).

2. J. Y. Huang, K. Kempa, S. H. Jo, S. Chen, and Z. F. Ren (submitted).

3. J.-M.Bonard, K.A. Dean, B.F. Coll, and C. Klinke, Phys. Rev.Lett. 89, 197602 (2002). 


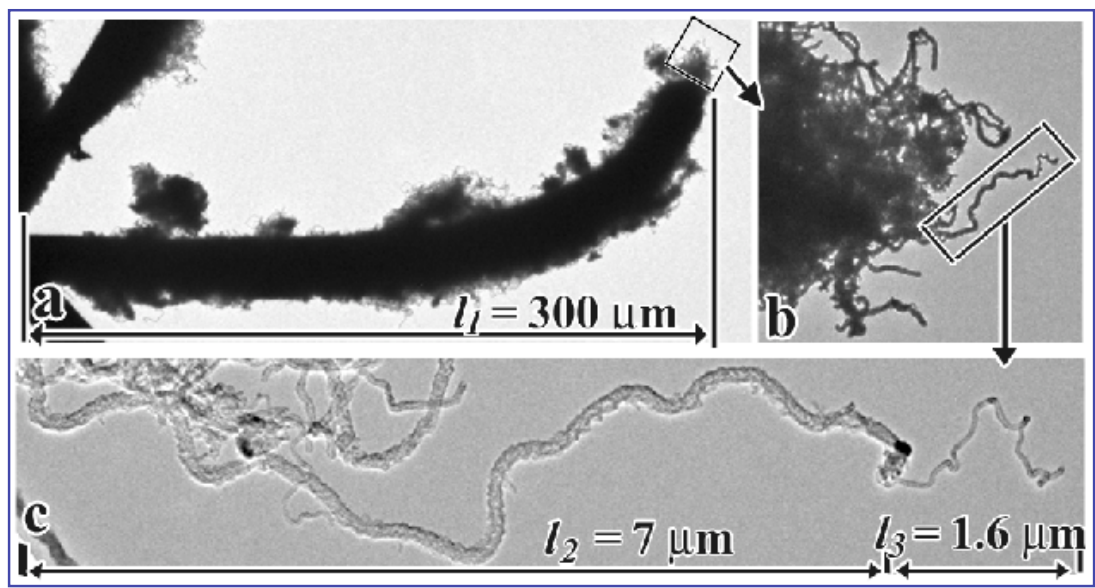

Fig. 1. Multi-stage growth geometry for nanotubes grown on a carbon fiber. (a), (b), and (c) are subsequent magnifications of the stages.
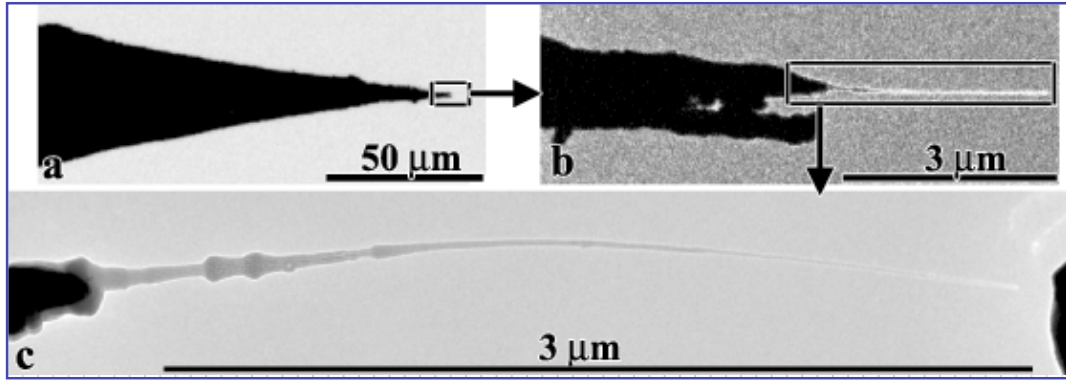

Fig. 2. Sequential TEM micrograph showing multi-stage geometry of a nanotube attached to the conical Au rod.
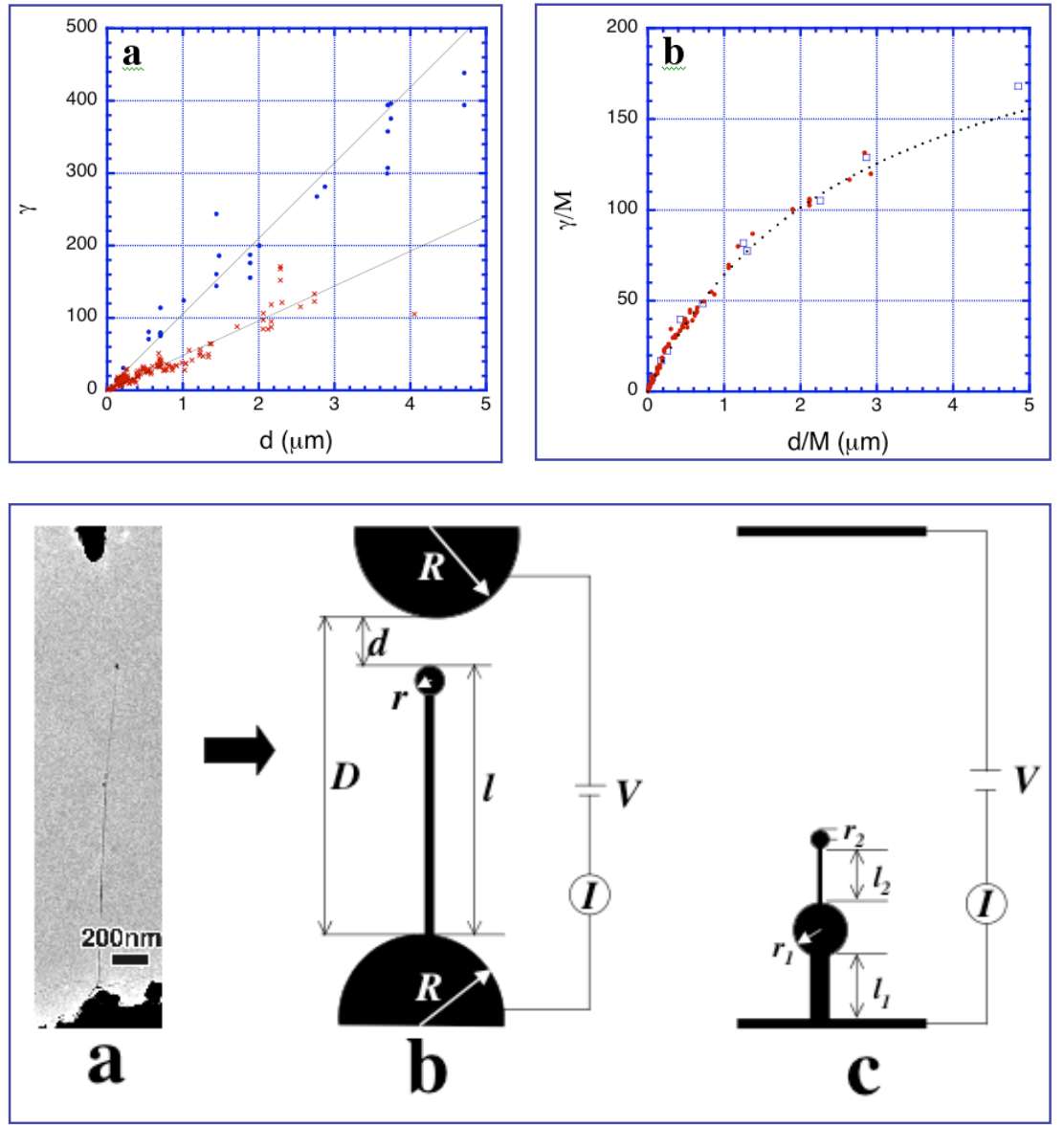

Fig. 3. $\gamma$ plots. (a) Small d regime, CNTs A $\bigcirc, \mathrm{B} \times$. The solid lines are interpolations of Eq. 2. (b) Large d regime for CNTs $\mathrm{A} \bigcirc$ and $\mathrm{C} \square$ from Ref. 3 . Dotted line represents a fit, by using Eq. 3.

Fig. 4. (a). A setup for field emission study, with a nanotube attached to a cathode (bottom). (b) A model of the experimental configuration shown in (a), used to calculate the electric field. (c) A simple model of a two-stage emitter. 